\title{
Expression levels of heat shock protein 20 decrease in parallel with tumor progression in patients with hepatocellular carcinoma
}

\author{
TAKAHIRO NODA $^{1,2}$, TAKASHI KUMADA ${ }^{3}$, SHINJI TAKAI ${ }^{1}$, RIE MATSUSHIMA-NISHIWAKI $^{1}$, \\ NAOKI YOSHIMI $^{5}$, EISUKE YASUDA ${ }^{1,2}$, KANEFUSA KATO $^{6}$, HIDENORI TOYODA ${ }^{3}$, \\ YUJI KANEOKA ${ }^{4}$, AKIHIRO YAMAGUCHI ${ }^{4}$ and OSAMU KOZAWA ${ }^{1}$ \\ ${ }^{1}$ Department of Pharmacology, Gifu University Graduate School of Medicine, 1-1 Yanagido, Gifu 501-1194; \\ Departments of ${ }^{2}$ Imaging Diagnosis, ${ }^{3}$ Gastroenterology and ${ }^{4}$ Surgery, Ogaki Municipal Hospital, \\ 4-86 Minaminokawa-Cho, Ogaki, Gifu 503-8502; ${ }^{5}$ Tumor Pathology, University of the Ryukyus \\ Faculty of Medicine, 207 Nishihara-Cho, Okinawa 903-0215; ${ }^{6}$ Department of Biochemistry, \\ Institute for Developmental Research, Aichi Human Service Center, \\ 713-8 Kamiya-Cho, Kasugai, Aichi 480-0392, Japan
}

Received December 12, 2006; Accepted February 14, 2007

\begin{abstract}
Heat shock protein (HSP) 20, a low-molecularweight HSP, is constitutively expressed in various tissues, such as smooth muscle, skeletal muscle, and liver. However, the characteristics and function of HSP20 have not been precisely understood. In the present study, we investigated correlations of expression levels of HSP20 in hepatocellular carcinoma (HCC) tissues and the surrounding tissues with clinical and pathologic characteristics in 53 resected HCC specimens. Although HSP20 was detected in all 53 HCC tissues, the expression levels were reduced compared with those in the adjacent non-tumor tissues. The expression levels of HSP20 were inversely correlated with tumor stage by TNM classification ( $\mathrm{p}<0.01)$, presence of microvascular invasion $(\mathrm{p}<0.05)$, and tumor size $(\mathrm{p}<0.05)$. Our findings strongly suggest that HSP20 may play a role against the progression of human HCC.
\end{abstract}

\section{Introduction}

Cells produce heat shock proteins (HSPs), when exposed to various kinds of biological stress such as heat and chemicals (1). HSPs are classified into high-molecular-weight HSPs

Correspondence to: Dr Osamu Kozawa, Department of Pharmacology, Gifu University Graduate School of Medicine, 1-1 Yanagido, Gifu 501-1194, Japan

E-mail: okozawa@gifu-u.ac.jp

Key words: heat shock protein 20, hepatocellular carcinoma, tumor stage such as HSP70, HSP90 and HSP110, and low-molecularweight HSPs with molecular masses from 10-30 kDa such as HSP20, HSP27 and $\alpha \mathrm{B}$-crystallin according to apparent molecular sizes. It is well recognized that high-molecularweight HSPs act as molecular chaperones in protein folding, oligomerization and translocation (1). Though the functions of low-molecular-weight HSPs are not as well characterized as those of the high-molecular-weight HSPs, it is recognized that they may also have chaperone functions (1). The human genome codes for 10 low-molecular-weight HSPs (2). In their C-terminal half, these proteins share a sequence element of $\sim 100$ amino acid residues called the $\alpha$-crystallin domain, and toward their $\mathrm{N}$-terminal end, they share a less conserved but nevertheless similar domain (3). HSP20 was co-purified with HSP27 and $\alpha \mathrm{B}$-crystallin from skeletal muscle, and it was identified as a member of the crystallin family (4). Although HSP20 is not induced by heat or chemical stress, it is highly expressed in normal skeletal and smooth muscle, heart and liver tissues where it may be essential, but the exact role of HSP20 remains to be clarified (4).

Hepatocellular carcinoma (HCC) is a common malignancy worldwide, and it causes more than one million deaths annually $(5,6)$. Factors that indicate tumor progression in association with patient outcome reportedly include tumor size, number of tumors, vascular invasion that can be evaluated pathologically and imaging diagnosis (7-10). Tumor markers for HCC such as $\alpha$-fetoprotein levels and des- $\gamma$-carboxy prothrombin levels are reported to be additional indicators of tumor progression associated with patient survival (10-13). However, these factors are not sufficient to accurately discriminate the tumor progression of HCC patients towards the accurate prediction of patient survival. It is, therefore, necessary to further investigate other indicators for the evaluation of tumor progression and for the prediction of patient outcome. 
To date, it has been reported that expression of certain HSPs can be correlated with the carcinogenic process as well as with the degree of differentiation and cell proliferation, and moreover, they have been implicated in the regulation of apoptosis $(14,15)$. In addition, evidence is accumulating about the usefulness of the prognostic implications of HSPs in certain cancer types, especially high-molecular-weight HSPs $(14,15)$. We have recently shown that attenuated phosphorylation of HSP27 correlates with tumor progression in patients with HCC (16). Among low-molecular-weight HSPs, HSP27 has been the most extensively studied, but to the best of our knowledge there has been no report about the relationship of HSP20 and tumor progression. Therefore, in the present study, we tried to investigate the relationship between HSP20 and $\mathrm{HCC}$ in 53 resected HCC specimens.

\section{Materials and methods}

Patients. Fifty-three patients (46 men, 7 women, mean age: $66.9 \pm 8.4$ years), having been diagnosed with HCC at the Department of Gastroenterology, Ogaki Municipal Hospital, Ogaki, Japan underwent hepatic resection between September 2002 and August 2005. Liver cirrhosis was present in 24 patients, and chronic hepatitis was present in 29. Fourteen patients were infected with hepatitis $\mathrm{B}$ virus, and 34 were infected with hepatitis $C$ virus. The remaining 5 patients had evidence of alcoholic cirrhosis. No patient had previously undergone preoperative chemotherapy.
The resected HCC specimens were obtained according to protocol approved by the Committee for the Conduct of Human Research at Ogaki Municipal Hospital. Informed consent was obtained from all patients.

Surgical specimens. Primary HCC tissues were obtained from all patients by surgical resection at the Department of Surgery, Ogaki Municipal Hospital. The excised tissue was divided into two parts, and one part was fixed with $20 \%$ neutral formalin overnight. The fixed tissue was then dehydrated with $100 \%$ methanol and xylene and embedded in paraffin wax. A threemicron-thickness of this tissue was used for immunohistochemical staining. The other part of the resected tissue was snap-frozen in liquid nitrogen and stored at $-80^{\circ} \mathrm{C}$ until used for Western blot analysis.

Pathological evaluations. The pathological features of HCC were evaluated by two of the authors (N.Y. and Y.K.) without knowledge of the HSP20 status of the tumor. The specimen was stained with hematoxylin and eosin, and the entire specimen was examined. Differentiation of HCC was classified as well-, moderately, or poorly differentiated HCC on the basis of the classification by the International Working Party (17). Vascular invasion and infiltration to the tumor capsule were evaluated macroscopically.

Western blot analysis. Snap-frozen samples were homogenized and sonicated in lysis buffer containing $62.5 \mathrm{mM}$ Tris- $\mathrm{HCl}$

Table I. Comparison of the protein levels of HSP20 with the clinical and pathological characteristics of 53 patients with HCC.

$\mathrm{p}$ value

$\overline{\text { Tumor tissue }} \quad$ Non-tumor tissue

Gender

male $(n=46)$, female $(n=7)$

Underlying disease

liver cirrhosis $(n=25)$, chronic hepatitis $(n=28)$

$0.010^{\mathrm{a}}$

Etiology of liver disease

$\operatorname{HBV}(n=13), \operatorname{HCV}(n=35)$, alcoholic $(n=5)$

0.662

0.482

Number of tumors

solitary $(n=40)$, multiple $(n=13)$

0.374

Tumor size $(\mathrm{mm})$ $<20(\mathrm{n}=12), 20-50(\mathrm{n}=32),>50(\mathrm{n}=9)$

Vascular invasion negative $(n=35)$, positive $(n=18)$

Infiltration to capsule negative $(n=28)$, positive $(n=25)$

Tumor stage

I ( $\mathrm{n}=9)$, II $(\mathrm{n}=25)$, III $(\mathrm{n}=11)$, IV $(\mathrm{n}=8)$

Histological classification (differentiation)

well- $(n=11)$, moderately $(n=35)$, poorly $(n=7)$

0.858

0.636

$\mathrm{HBV}$, hepatitis B virus; HCV, hepatitis C virus; ${ }^{\mathrm{a}} \mathrm{p}<0.05$. 


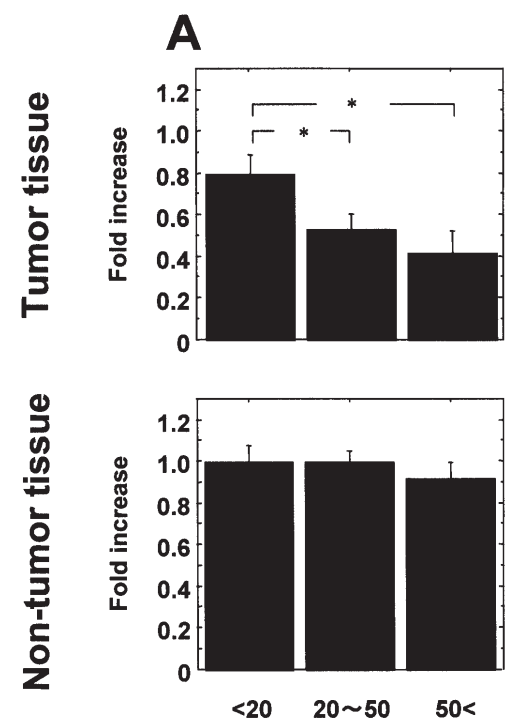

Tumor size (mm)

\section{B}
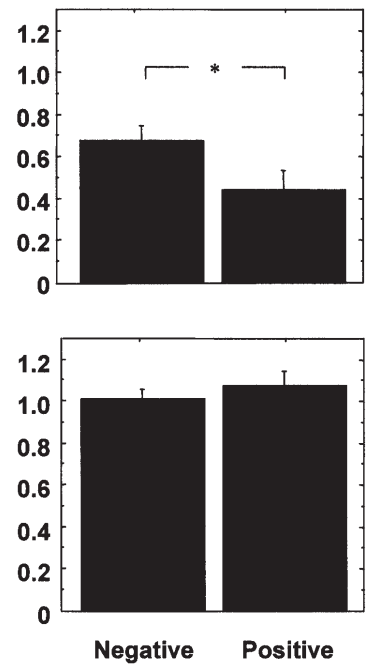

Vascular invasion
C
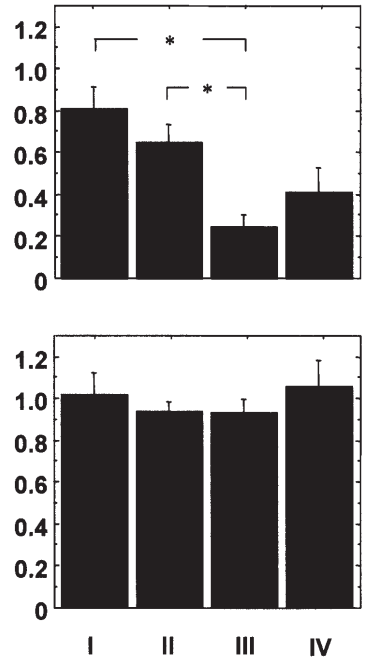

Tumor stage

Figure 1. HSP20 levels in patients with HCC. Protein extracts from 53 HCC specimens (tumor and adjacent non-tumor tissue, respectively) were analyzed with antibodies against HSP20 and B-actin. Signal intensities on X-ray film were quantified with NIH image software. The histograms show quantitative representations of the levels of HSP20 after normalization to levels of $\beta$-actin. Values on the vertical axis represent the mean \pm SE of independent experiments. The values were calculated as the average values with those of small (tumor size $<20 \mathrm{~mm}$ ) HCC (left column), negative vascular invasion (center column) and tumor stage I HCC (right column) equal to 1.0 . ${ }^{*} \mathrm{p}<0.05$.

( $\mathrm{pH} 6.8$ ), $2 \%$ sodium dodecyl sulfate (SDS), $50 \mathrm{mM}$ dithiothreitol, and $10 \%$ glycerol. Western blot analysis was performed as described previously (18) with polyclonal antibodies against HSP20 and HSP27 (Stressgen Biotechnologies, Victoria, British Columbia, Canada). Peroxidase-conjugated antibodies against rabbit $\operatorname{IgG}$ were used as secondary antibodies against the above-mentioned primary antibodies. Primary antibodies against $\beta$-actin (Sigma-Aldrich Co, St. Louis, MO) were detected with peroxidase-conjugated antibodies against mouse IgG as secondary antibodies. Peroxidase activity on PVDF membranes was visualized on X-ray film with the ECL Western blotting detection system (GE Healthcare UK Ltd, Buckinghamshire, UK). Protein band intensities were determined by integrating the optical density over the band area (band volume) with NIH image software. HSP20 levels were normalized to those of $\beta$-actin.

Immunohistochemical analysis. Immunohistochemical staining of some specimens was performed with the streptavidinbiotin complex method to investigate expression and localization of HSP20. Primary antibodies were anti-HSP20 rabbit polyclonal antibodies (Stressgen Biotechnologies, Golden, $\mathrm{CO})$. Briefly, deparaffinized sections were treated with $3 \%$ $\mathrm{H}_{2} \mathrm{O}_{2}$ in methanol for 10 min to inhibit endogenous peroxidase activity. Sections were immersed in $0.05 \mathrm{M}$ citrate buffer ( $\mathrm{pH}$ 6.0), heated in a microwave oven for $15 \mathrm{~min}$, and then incubated with primary antibodies for $2 \mathrm{~h}$ at room temperature. Each section was treated sequentially with biotinylated secondary antibodies (anti-rabbit-IgG) and streptavidinperoxidase complex (Dako Chem Mate, Kyoto, Japan). Finally, immune complexes were visualized with 3,3'-diaminobenzidine tetrahydrochloride as a chromogen. Mayer's hematoxylin was used as a counterstain.
Statistical analysis. Patient clinical data were expressed as mean \pm SD. The data were analyzed with the SPSS software program (Release 11.5.1J standard version; SPSS Japan, Tokyo, Japan). One-way analysis of variance (ANOVA) was used to determine the significance of differences between protein expression and grade of tumor differentiation or tumor stage. Nonparametric data were analyzed with the Mann-Whitney U test, Kruskal-Wallis test, or Spearman's correlation coefficient (r). All p values were derived from two-tailed tests and $\mathrm{p}<0.05$ was accepted as statistically significant. A Spearman's correlation coefficient of $r \geq 0.400$ was accepted as a positive correlation.

\section{Results}

Correlations of HSP2O levels according to characteristics of $H C C$. The levels of HSP20 were compared with the clinical and pathological characteristics of 53 patients with HCC, including gender, underlying liver disease, etiology, number of tumors, tumor size, vascular invasion, infiltration to the tumor capsule, and tumor stage (evaluated according to the TNM classification of the International Union Against Cancer) (19), and histological classification (Table I). Comparisons of the levels of HSP20 revealed significant differences with respect to tumor size $(\mathrm{p}=0.048)$, vascular invasion $(\mathrm{p}=0.040)$ and tumor stage $(\mathrm{p}=0.003)$ in tumor tissues, while there were no significant differences in HSP20 levels in adjacent nontumor tissues, except in those tissues with underlying liver disease (Table I). In the non-tumor tissues, the levels of HSP20 in liver cirrhotic tissue were significantly higher than those in chronic hepatitis patient tissue (Table I).

HSP20 levels according to tumor size, vascular invasion and tumor stage are shown in Fig. 1. A trend toward decreased expression levels of HSP20 in tumor tissues was 


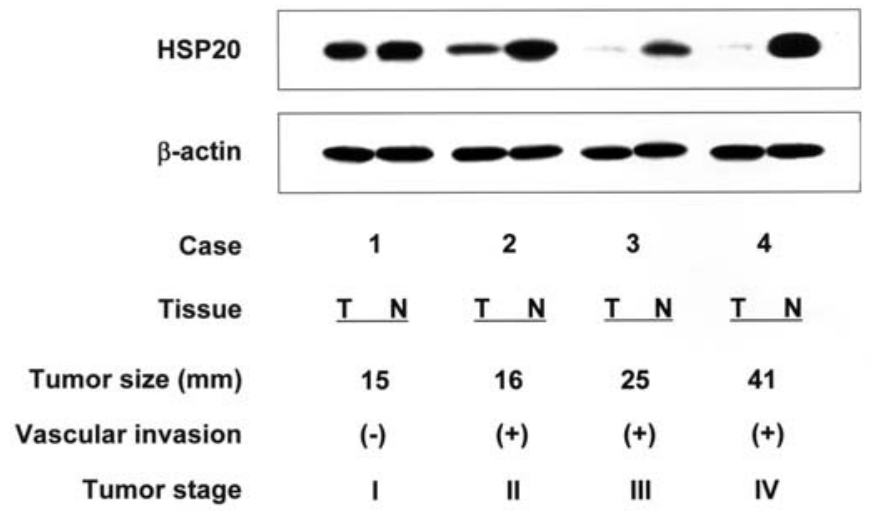

Figure 2. HSP20 levels in four representative patients with HCC according to tumor size, vascular invasion and tumor stage. Comparison with those in non-tumor tissue. Protein extracts were analyzed with antibodies against HSP20 and B-actin. T, tumor tissue; $\mathrm{N}$, adjacent non-tumor tissue.

observed as tumor size, vascular invasion and tumor stage increased, suggesting that the levels of HSP20 in the adjacent non-tumor tissues were higher than those in the tumor tissues (Fig. 1A, B and C; upper panel). On the other hand, HSP20 levels in the adjacent non-tumor tissues were not correlated with these factors, suggesting that the levels of HSP20 in the tumor tissues were attenuated in parallel with $\mathrm{HCC}$ progression (Fig. 1A, B and C; lower panel). Western blot images of HSP20 expression in 4 representative patients with HCC according to tumor size, vascular invasion and tumor stage are shown in Fig. 2.

Immunohistochemical analysis of HSP2O in HCC specimens. To confirm our results from Western blot analysis, we performed immunohistochemical analysis of HSP20 in HCC tumor and non-tumor tissues. Immunohistochemical staining of HSP20 in stage-IV-HCC specimens containing tumor and non-tumor tissue is shown in Fig. 3. Immunoreactivity for HSP20 in tumor tissue was markedly lower than that in nontumor tissue.

Comparisons between the levels of HSP2O and the levels of phosphorylated HSP27 in HCC tumor tissues. HSP27, a low- molecular-weight HSP, is phosphorylated at three serine residues (Ser-15, Ser-78 and Ser-82) (1). We previously reported that attenuation of phosphorylated HSP27 (Ser-15, Ser-78 and Ser-82) in tumor tissue correlates with HCC progression (16). Therefore, we investigated the correlation between the levels of HSP20 and the levels of phosphorylated HSP27 that had been determined in the previous study. The levels of phosphorylated HSP27 (Ser-15) were significantly correlated with the levels of HSP20 ( $r=0.505, p<0.001$; Fig. 4A). On the contrary, the levels of phosphorylated HSP27 (Ser-78), phosphorylated HSP27 (Ser-82) or total HSP27 were not correlated with those of HSP20 (Fig. 4B, C and $\mathrm{D}$, respectively).

\section{Discussion}

In the present study, we showed that attenuation of HSP20 levels correlated with tumor progression in tumor tissues of patients with HCC. In addition, the HSP20 levels correlated inversely with tumor size and vascular invasion of HCC, both of which are indications of an advanced tumor. To the best of our knowledge, this is the first report of a significant relation between HSP20 levels and progression of HCC.

Recently, we reported that attenuation of phosphorylated HSP27 is correlated with HCC progression (16). It is recognized that $\mathrm{HSP} 27$, $\mathrm{HSP} 20$ and $\alpha \mathrm{B}$-crystallin form one type of complex $(3,20)$. It has been shown that phosphorylation of HSP27 is associated with the disassembly of HSP27 complexes $(21,22)$. In the present study, we found significant correlation between the levels of HSP20 and that of phosphorylated HSP27 (Ser-15), but not Ser-78 and Ser-82. Although the differential role of the three phosphorylation sites are not known, our findings suggest that HSP20 and phosphorylated HSP27 (Ser-15) may have suppressive effects on HCC progression. In addition, these results suggest that phosphorylated HSP27 (Ser-78) and phosphorylated HSP27 (Ser-82) may have different roles in HCC progression. Further investigations are required not only to clarify the exact role of HSP20, but also to determine whether these HSPs can be prognostic factors in HCC. Moreover, HSPs not only have prognostic implications but also have therapeutic implications for cancer (14). Among HSPs, the use of the HSP90 inhibitor,
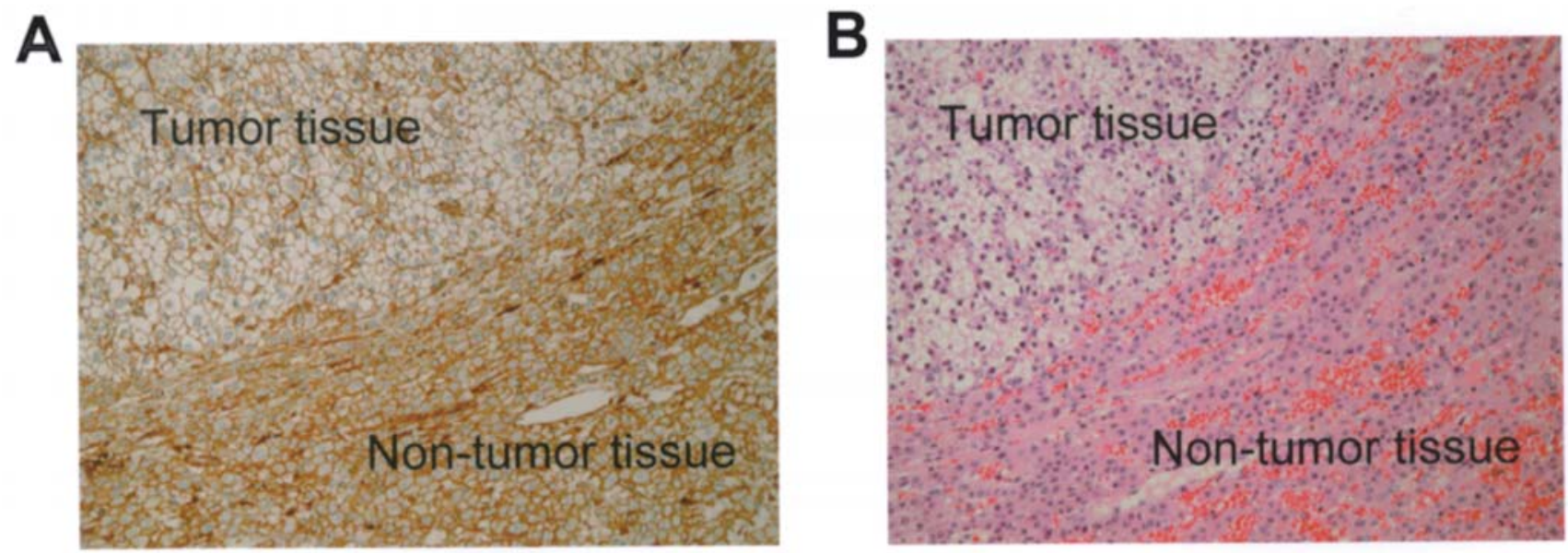

Figure 3. Immunohistochemical analysis of HSP20 in a patient with HCC (tumor stage IV) and adjacent non-tumor tissue (chronic hepatitis) (A). The same patient specimen stained with hematoxylin and eosin. (B). 
A

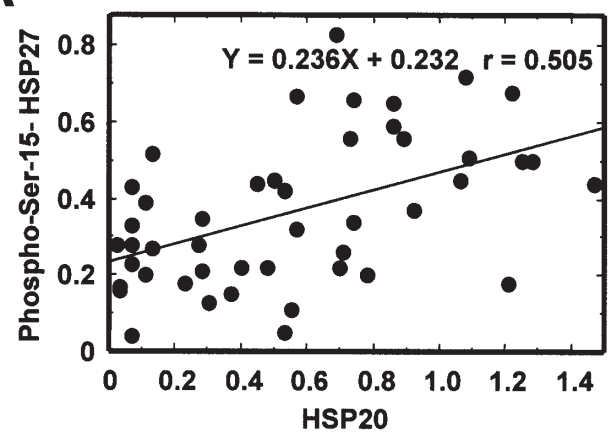

C

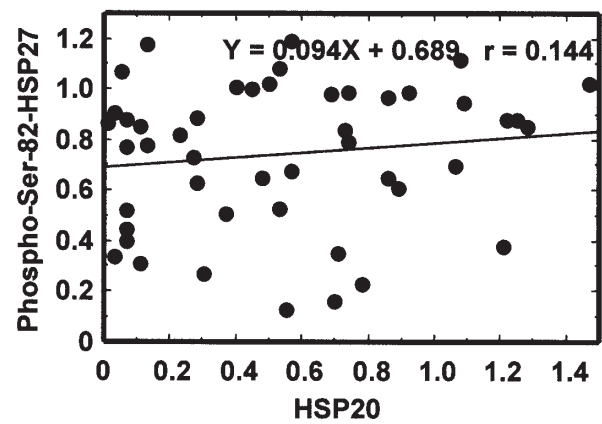

B

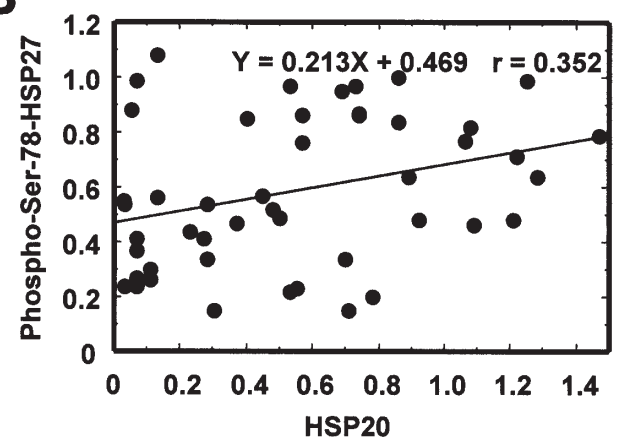

D

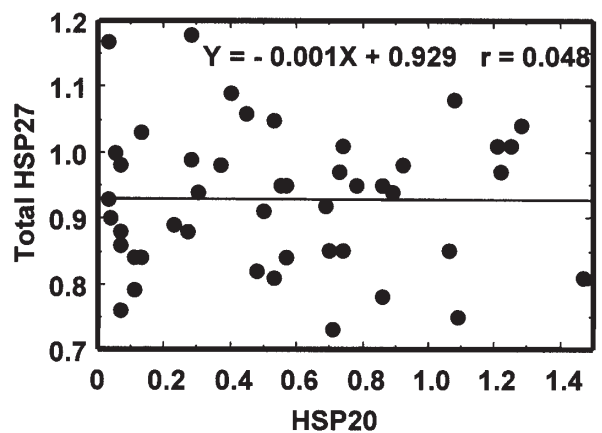

Figure 4. Correlations between the levels of HSP20 and the levels of (A) Ser-15-phosphorylated HSP27, (B) Ser-78-phosphorylated HSP27, (C) Ser-82phosphorylated HSP27, and (D) total HSP27. The expression levels of HSP20, phosphorylated HSP27, and total HSP27 were determined by the band intensities obtained from Western blot analysis, and then normalized to those of $\beta$-actin.

which is under phase I trial has been extensively studied $(14,15)$. Although the role of HSP20 in HCC is not precisely known, further investigations would help us to use HSP20 as a target for cancer therapy.

In conclusion, our present results strongly suggest that expression levels of HSP20 decrease with progression in tumor stages in patients with HCC and that HSP20 may have a suppressive effect on the advancement of human HCC.

\section{Acknowledgements}

This study is supported in part by the Japanese Clinical Oncology Fund.

\section{References}

1. Benjamin IJ and McMillan DR: Stress (heat shock) proteins: molecular chaperones in cardiovascular biology and disease. Circ Res 83: 117-132, 1998.

2. Taylor RP and Benjamin IJ: Small heat shock proteins: a new classification scheme in mammals. J Mol Cell Cardiol 38: 433-444, 2005.

3. Sun X, Welsh MJ and Benndorf R: Conformational changes resulting from pseudophosphorylation of mammalian small heat shock proteins - a two-hybrid study. Cell Stress Chaperones 11: 61-70, 2006.

4. Kato K, Goto S, Inaguma Y, Hasegawa K, Morishita R and Asano T: Purification and characterization of a $20-\mathrm{kDa}$ protein that is highly homologous to alpha B crystallin. J Biol Chem 269: 15302-15309, 1994.

5. Bosch FX, Ribes J and Borras J: Epidemiology of primary liver cancer. Semin Liver Dis 19: 271-285, 1999.

6. Montesano R, Hainaut P and Wild CP: Hepatocellular carcinoma: from gene to public health. J Natl Cancer Inst 89: 1844-1851, 1997.
7. Izumi R, Shimizu K, Tohru I, Yagi M, Matsui O, Nonomura A and Miyazaki I: Prognostic factors of hepatocellular carcinoma in patients undergoing hepatic resection. Gastroenterology 106: 720-727, 1994.

8. Okada S, Shimada K, Yamanaka J, Takayama T, Kosuge T, Yamasaki S, Sakamoto M and Hirohashi S: Predictive factors for postoperative recurrence of hepatocellular carcinoma. Gastroenterology 106: 1618-1624, 1994

9. Shimada M, Takenaka K, Taguchi K, Fujiwara Y, Kajiyama K, Maeda T, Shirabe K, Yanaga K and Sugimachi K: Prognostic factors after repeat hepatectomy for recurrent hepatocellular carcinoma. Ann Surg 227: 80-85, 1998.

10. Koda M, Murawaki Y, Mitsuda A, Ohyama K, Horie Y, Suou T, Kawasaki $\mathrm{H}$ and Ikawa S: Predictive factors for intrahepatic recurrence after percutaneous ethanol injection therapy for small hepatocellular carcinoma. Cancer 88: 529-537, 2000.

11. Oka H, Tamori A, Kuroki T, Kobayashi K and Yamamoto S: Prospective study of alpha-fetoprotein in cirrhotic patients monitored for development of hepatocellular carcinoma. Hepatology 19: 61-66, 1994.

12. Kuromatsu R, Tanaka M, Shimauchi Y, Shimada M, Tanikawa K, Watanabe K and Yokoo T: Usefulness of ED036 kit for measuring serum PIVKA-II levels in small hepatocellular carcinoma. J Gastroenterol 32: 507-512, 1997.

13. Mita Y, Aoyagi Y, Yanagi M, Suda T, Suzuki Y and Asakura H: The usefulness of determining des- $\gamma$-carboxyl prothrombin by sensitive enzyme immunoassay in the early diagnosis of patients with hepatocellular carcinoma. Cancer 82: 1643-1648, 1998

14. Ciocca DR and Calderwood SK: Heat shock proteins in cancer: diagnostic, prognostic, predictive, and treatment implications. Cell Stress Chaperones 10: 86-103, 2005.

15. Calderwood SK, Khaleque MA, Sawyer DB and Ciocca DR: Heat shock proteins in cancer: chaperones of tumorigenesis Trends Biochem Sci 31: 164-172, 2006.

16. Yasuda E, Kumada T, Takai S, Ishisaki A, Noda T, Matsushima-Nishiwaki R, Yoshimi N, Kato K, Toyoda H, Kaneoka Y, Yamaguchi A and Kozawa O: Attenuated phosphorylation of heat shock protein 27 correlates with tumor progression in patients with hepatocellular carcinoma. Biochem Biophys Res Commun 337: 337-342, 2005. 
17. International Working Party: Terminology of nodular hepatocellular lesions. Hepatology 22: 983-993, 1995.

18. Kato K, Ito $\mathrm{H}$, Hasegawa $\mathrm{K}$, Inaguma $\mathrm{Y}$, Kozawa $\mathrm{O}$ and Asano $\mathrm{T}$ : Modulation of the stress-induced synthesis of hsp27 and $\alpha \mathrm{B}$ crystallin by cyclic AMP in C6 rat glioma cells. J Neurochem 66: 946-950, 1996

19. International Union Against Cancer (UICC): TNM Classification of Malignant Tumors. Sobin LH and Wittekind C (eds). WileyLiss Inc., New York, pp74-77, 1997.

20. Sugiyama Y, Suzuki A, Kishikawa M, Akutsu R, Hirose T, Waye MM, Tsui SK, Yoshida S and Ohno S: Muscle develops a specific form of small heat shock protein complex composed of MKBP/HSPB2 and HSPB3 during myogenic differentiation. J Biol Chem 275: 1095-1104, 2000.
21. Kato K, Ito H, Iwamoto I, Lida K and Inaguma Y: Protein kinase inhibitors can suppress stress-induced dissociation of HSP27. Cell Stress Chaperones 6: 16-20, 2001

22. Hirano S, Sun X, Deguzman CA, Ransom RF, McLeish KR, Smoyer WE, Shelden EA, Welsh MJ and Benndorf R: p38MAPK/HSP25 signaling mediated cadmium-induced contraction of mesangial cells and renal glomeruli. Am J Physiol Renal Physiol 288: 1133-1143, 2005. 